\title{
Chamanismes de l'Asie de l'Est. Termes et rôles en mouvement
}

À propos de :

Hu Tai-li, LIU, Pi-chen (dir.), Taiwan yuanzhumin wushi yuyishi zhanbian (Chamanisme et performances rituelles des aborigènes de Taiwan), Taipei, Academia Sinica, Institut d'ethnologie, 2010, 569 p.

KISTER Daniel A., "Shamanic Worlds of Korea and Norhteast Asia », Séoul, Jimoondang, Korean Studies Series, $\mathrm{n}^{\circ} 45,2010,324 \mathrm{p}$.

LIU Pi-chen, Rentong, xingbie yu juluo : gamalan ren bianqian zhong de yishi yanjiu (Identité, sexe et localité : recherche sur les transformations rituelles chez les Kavalan), Nantou, Taiwan Historica, 2008, 400 p. RUDOLPH Michael, Ritual Performances as Authenticating Practices, Cultural Representations of Taiwan's Aborigines in Times of Political Changes, Berlin, LIT Verlag, «Performanzen », vol. 14, 2008, 269 p. ZHAO Zhizhong, Zhongguo samanjiao (Le chamanisme chinois), Xining, Presses populaires du Qinghai, 2008, $241 \mathrm{p}$.

\section{Benoît Vermander}

\section{(2) OpenEdition}

Édition électronique

URL : http://journals.openedition.org/assr/24524

DOI : $10.4000 /$ assr. 24524

ISSN : $1777-5825$

Éditeur

Éditions de l'EHESS

Édition imprimée

Date de publication : 30 décembre 2012

Pagination : 35-61

ISSN : 0335-5985

Référence électronique

Benoît Vermander, «Chamanismes de l'Asie de l'Est. Termes et rôles en mouvement », Archives de sciences sociales des religions [En ligne], 160 | octobre-décembre 2012, mis en ligne le 20 février 2016, consulté le 20 avril 2019. URL : http://journals.openedition.org/assr/24524 ; DOI : 10.4000/assr.24524 


\title{
Benoît Vermander
}

\section{Chamanismes de l'Asie de l'Est}

\section{Termes et rôles en mouvement}

\author{
À propos de : \\ Hu Tai-li, Liu, Pi-chen (dir.), Taiwan yuanzhumin wushi yuyishi zhan- \\ bian (Chamanisme et performances rituelles des aborigènes de \\ Taiwan), Taipei, Academia Sinica, Institut d'ethnologie, 2010, \\ $569 \mathrm{p}$. \\ KISTER Daniel A., "Shamanic Worlds of Korea and Norhteast Asia », \\ Séoul, Jimoondang, Korean Studies Series, $\mathrm{n}^{\circ}$ 45, 2010, 324 p. \\ LIU Pi-chen, Rentong, xingbie yu juluo : gamalan ren bianqian zhong \\ de yishi yanjiu (Identité, sexe et localité : recherche sur les trans- \\ formations rituelles chez les Kavalan), Nantou, Taiwan Historica, \\ 2008, $400 \mathrm{p}$. \\ Rudolph Michael, Ritual Performances as Authenticating Practices, \\ Cultural Representations of Taiwan's Aborigines in Times of Poli- \\ tical Changes, Berlin, LIT Verlag, " Performanzen », vol. 14, 2008, \\ $269 \mathrm{p}$. \\ ZHAO Zhizhong, Zhongguo samanjiao (Le chamanisme chinois), \\ Xining, Presses populaires du Qinghai, 2008, 241 p.
}

S'il est un champ que l'anthropologie des religions a parcouru tout à la fois avec constance et une conscience critique perpétuellement en éveil, c'est bien celui du "chamanisme». Constance et "mauvaise conscience» associées que suscitait peut-être l'avertissement - souvent répété par la suite - qu'Arnold Van Gennep adressait aux ethnographes : « La terminologie de la science des religions est encore si peu fixée, les notions dont elle traite sont tellement complexes et leur étude si peu débrouillée qu'il est nécessaire de n'employer chaque mot destiné à désigner un ensemble de coutumes et de croyances qu'en lui donnant le sens le plus exact possible. [...] Parmi ces mots vagues, l'un des plus dangereux est celui de chamanisme. » (Van Gennep, 1903, p. 51). Or, il se trouve que les ouvrages et articles dont j'entends rendre compte ici, tous consacrés à l'Asie du Nord-Est ou du Sud-Est, ne font pas usage des mêmes précautions méthodologiques ou terminologiques que celles qui ont marqué d'autres moments et d'autres lieux 
de la recherche. Non pas qu'ils donneraient au terme de " chamanisme » l'usage englobant et quasi polémologique que l'on a pu aussi connaître, mais ils privilégient un usage évolutif et suggestif de la notion : au parti-pris étroitement définitionnel de Van Gennep, aux tentatives de circonscrire un phénomène par nature souple et ondoyant a succédé une stratégie méthodologique qui confère au mot employé un espace mouvant et pourtant structuré par son association avec des concepts et récits appelés par l'objet d'étude. Il s'agit donc pour nous tout à la fois de rendre compte de l'application d'une notion donnée à des champs géographiques où le terme a joué et joue toujours un rôle important et de nous interroger sur ce que l'évolution même de son usage peut nous enseigner.

On sait qu'à partir des années 1980, s'est mise en place une compréhension nouvelle de la notion : la plasticité du chamanisme, survivant et se modifiant au travers des évolutions culturelles, sociales, économiques et religieuses, s'explique par la spécificité de ce qu'il offre : un système de gestion de l'aléatoire au travers d'un mécanisme d'alliance. Aussi, " toujours présent aux marges d'autres idéologies, le chamanisme est même revivifié par la situation de crise, car il offre le recours de la connivence personnelle avec des partenaires indéfiniment renouvelables : il ouvre la voie de la chance" (Hamayon, 1998a, p. 306). Si le thème de la «voyance " produite par la "transe » était central dans l'approche d'Eliade, c'est celui de "l'alliance » qui devient dominant dans les travaux d'Hamayon: le chamane gère la circulation de l'énergie vitale entre les âmes des espèces animales et celles du genre humain et, pour ce faire, il conclut une alliance matrimoniale avec la surnature, les schémas matrimoniaux possibles restant multiples. Le chamane devient habituellement le gendre de la surnature en épousant la fille de l'Esprit pourvoyeur de subsistance animale. Dans les sociétés où se produit une féminisation du chamane, les femmes chamanes ne peuvent s'allier directement avec un Esprit mais concluront alliance avec l'âme d'un défunt (Hamayon, 1990b, p. 41-42). Par ailleurs, le caractère ambigu ou indifférencié du sexe du chamane, caractérisé par une sorte de bisexualité, tant pour les hommes que pour les femmes, a été souvent relevé par la littérature (Vazeilles, 1990, p. 182 ; Métraux, 1967, p. 181 ; Boyer-Araujo, 1993, p. 147-151 ; Saladin d'Anglure, 1986 et 1992). Pour certains, l'ambiguïté de la sexualité du chamane est liée au caractère fondamentalement androgyne de ses alliés, les esprits (Hell, 1999, p. 261-270).

\section{Wu et chamanes}

Il existe une difficulté inhérente à l'usage du terme de chamane : faut-il en faire emploi seulement pour l'aire géographique où ce terme (ou un terme étymologiquement voisin) est attesté, ou faut-il l'utiliser pour désigner une classe de praticiens rituels, indépendamment des termes locaux habituellement en usage ?

Dans le contexte culturel chinois, cette question se ramène au choix à effectuer entre les termes de wushi et de saman. La solution habituellement retenue 
consiste à utiliser le terme de wushi huodong (activités du wu, activités médiumniques) en chinois et à le traduire systématiquement par shamanism en anglais... et réciproquement. Ainsi, le livre dirigé par $\mathrm{Hu}$ Tai-li et Liu Pi-chen intitulé Taiwan yuanzhumin wushi yu yishi zhanyan est-il traduit par Shamans and Ritual Performances of the Indigenous Peoples in Taiwan dans le titre anglais qu'il offre sur sa quatrième de couverture (Hu Tai-li \& Liu Pi-chen, 2010). L'équivalence ainsi proposée entre $w u$ et shaman remonte à Hopkins, Shafer et Walley (Hopkins, 1945 ; Shafer, 1951 ; Waley, 1955). Les questions ouvertes par cet usage sont multiples (Keightley, 1998, p. 829-831; Boileau, 2002). Mentionnons-en seulement trois: (a) le terme de wu ou wushi, spécifique au monde Han, est-il vraiment plus approprié pour rendre compte des activités rituelles des populations aborigènes de Taiwan, de souche austronésienne, que celui de saman, lequel a au moins le mérite de décentrer l'approche ethnographique de celle utilisée pour rendre compte de l'activité des jitong en contexte culturel han (jitong en mandarin, tâng-ki en hoklo : littéralement " jeune médium ", terme directement relié à la littérature ancienne sur les wu chinois - cf. Marshall, 2003) ? (b) De la même façon, l'usage systématique du terme wushi ne distordil pas la compréhension des pratiques rituelles d'autres minorités chinoises, celles du Sud-Ouest par exemple ? (c) À l'inverse, si le terme wushi s'avère avoir le mérite de mettre au jour une configuration culturelle et rituelle différente de celle ordinairement désignée par le terme de saman, ne faudrait-il pas alors trouver un équivalent anglais qui ne soit pas le mot shamanism? Mais pareilles questions risquent de devenir très vite scolastiques, et peut-être faut-il mieux accepter les usages courants, tout en sachant en rechercher les sources et justifier pragmatiquement les choix faits lorsque la question de leur pertinence est ouverte.

Comme souligné déjà par Paul Pelliot (Pelliot, 1913), l'usage du terme chamane (saman) en chinois est attesté d'ancienne date. Le troisième chapitre du Sanchao beimeng huibian, annales de la dynastie Song dues à Xu Mengxin (1124-1207) signale la réputation de chamane du Jurchen Wanyan Xiyin (m. 1140). Or, quoique le nom de ce personnage, réputé avoir conçu le premier système de transcription graphique de la langue jurchen, soit mentionné dans diverses sources, pas un seul épisode de sa vie ne semble lui attribuer des pouvoirs surnaturels. Il semble que le terme de "chamane " lui ait été conféré par le peuple en fonction de sa grande sagesse et habileté, lesquelles évoquaient les pouvoirs surnaturels des véritables chamanes (Kosa, 2007 : 116-118). Par ailleurs, le texte de Xu Mengxin identifie saman et wuyu (femme $w u$ ), ce qui est significatif du contexte historique de sa rédaction : la répression par l'État des $w u$ et des fangshi durant la dynastie Song, notamment à la toute fin $\mathrm{du} \mathrm{X}^{\mathrm{e}}$ siècle, a probablement réduit le registre de leurs activités et féminisé davantage encore la profession.

Le débat sur la relation entre le chamanisme et la religion chinoise archaïque remonte à deux célèbres articles, l'un de Tong Enzhen (Tong, 1930), qui tentait de concilier témoignages scripturaires et ethnographiques, l'autre de Chen Mengjia 
(Chen, 1936), lequel identifiait la religion Shang au wushu (invocations, divinations, danses pour la pluie), dont le roi exerçait - prétendait-il - la prêtrise en chef (cette vue a été reprise notamment par K. C. Chang, 1990 et 1994 ; Ching, $1997 \mathrm{a}$ et b). Les traits chamaniques des empereurs mythiques Huangdi, Yao et surtout $\mathrm{Yu}$, le caractère extatique qu'ont pu comporter les séances de divination royale et enfin certaines figurations des réceptacles sacrificiels en bronze sont les arguments les plus souvent invoqués à l'appui de cette thèse (voir aussi Paper, 1978 et 1995). L'article de Chen Mengjia a été critiqué de plusieurs points de vue (notamment du fait que, dans les inscriptions oraculaires, les caractères wang - roi - et $w u$ - chamane - ont toujours existé indépendamment), mais il jetait les bases de la recherche sur ce que l'on a appelé le «chamanisme Shang » (Chang, 1994 ; Johansen, 1987 ; Eichhorn, 1979). Chen Mengjia avait été fortement influencé dans sa recherche par les théories de Frazer, pour lequel le roi était d'abord un puissant sorcier. Ce qui nous intéresse ici, c'est que l'optique de Chen Mengjia qui lie fortement l'organisation politique et le culte religieux, est cela justement qui nous permet aujourd'hui d'établir - à son encontre - une distinction très nette entre le $w u$ et le chamane. Gilles Boileau a esquissé un parallèle critique entre le $w u$ de la Chine archaïque et le chamane de l'aire sibérienne: (a) le $w u$ de l'aire chinoise peut être mâle ou femelle et s'inscrit dans une organisation sociale très hiérarchisée ; le chamane sibérien est d'abord un mâle qui devient l'époux d'un esprit de la nature et il s'inscrit dans une "société sans État ", une société qui est fondée sur une coopération égalitaire - coopération entre les hommes qui composent le groupe, coopération entre le groupe pris collectivement et la nature; (b) le principal acte de la société religieuse chinoise archaïque est le sacrifice aux ancêtres, tandis que celui de la société sibérienne est la séance chamanique, sans sacrifice; (c) les principales fonctions du $w u$ chinois archaïque, pour autant qu'on puisse les établir, sont les exorcismes, les guérisons et la danse pour la pluie ; celles des chamanes sibériens ont à voir avec l'obtention du gibier et les guérisons ; (d) la voie d'accès à la fonction de $w u$ dans la société chinoise archaïque est inconnue; elle est souvent liée au choix du chamane effectué par un esprit dans l'aire sibérienne, et parfois au fait d'avoir un ancêtre chamane ; (e) le $w u$ chinois pratique ses rituels à l'extérieur, et souvent dans un lieu sauvage, tandis que le chamane sibérien les pratique ordinairement dans une hutte, ou en tout cas dans un lieu habité par les humains ; (f) en termes politiques, le $w u$ est associé au pouvoir, parfois comme officier du roi, ou bien quelquefois il est vu comme un ennemi politique de l'État, tandis que dans les sociétés de l'aire sibérienne le chamane est simplement situé en dehors de la structure politique (ou bien encore l'idée de structure politique autonome n'est-elle pas pertinente en pareil contexte) [Boileau, 2002, p. 376-378]. Si un parallèle entre le $w u$ chinois et l'aire sibérienne peut être esquissé, alors il doit l'être avec les parties du monde sibérien où la chasse a été supplantée par l'élevage : dans ces cultures, le sacrifice aux ancêtres est assuré par les anciens du clan, en vue de s'assurer la bienveillance continuée de la nature, et le chamane 
est réduit à un rôle subalterne. Les intervenants religieux plus élevés deviennent peu à peu les moines lamaïques, et la profession de chamane peut se féminiser (Hamayon, 1990a, p. 406-451). Bien entendu, ce parallèle est trop schématique, mais il inspire quelques observations : l'évolution politique de la Chine a très vite bouleversé le sens et le déroulement des pratiques rituelles de type chamanique, à supposer même (ce qui est très contestable) que, durant l'ère néolithique, le monde chinois et le monde sibérien aient partagé des rites et des croyances similaires (parmi les auteurs qui font l'hypothèse de l'existence d'une culture chamanique dans la Chine du néolithique, relevons: Chang, 1983, 1999, p. 50-53, dans une optique très marquée par Eliade ; Matthieu, 1987 ; Yang, 2005. Revue critique de certains de ces auteurs et d'autres in Keightley, 1998 et Kosa, 2000). En fin de compte, le $w u$ chinois semble constituer un type d'intervenant religieux très particulier, assez différencié du chamane, et qui pourrait peut-être se voir comparé à d'autres types religieux rencontrés en d'autres civilisations, non pas sur la base de l'idéal type chamanique, mais sur la base d'un autre idéal type qui reste à spécifier - à moins que justement la configuration politico-religieuse propre à la Chine ne soit cela même qui rende tout rapprochement difficile.

Le parallèle entre les inscriptions oraculaires et les textes parlant de l'époque Shang d'une part, les témoignages de l'époque Zhou de l'autre, complique encore l'analyse. Dans les inscriptions Shang, le caractère $w u$ désigne parfois un esprit, parfois un type de sacrifice lié au contrôle des vents, parfois peut-être un territoire et une divinité, parfois un être humain dont la fonction et la nature sont peu claires : cet être humain semble venir d'un autre territoire que celui du royaume, être offert en tribut au roi, et effectuer sur ordre certains sacrifices (Tu, 1997 ; Wang, 2001 ; Lefeuvre, 1997). Son statut et son rôle semblent avoir été assez limités. Les textes historiques qui présentent les $w u$ sous un jour assez favorable ne sont certainement pas à comprendre comme un portrait fidèle de l'ancienne civilisation chinoise, mais comme une construction idéale a posteriori : le Guoyu (compilation historique des $\mathrm{V}^{\mathrm{e}}$ et IV siècles av. J.-C.) insiste sur la nécessité de contrôler l'ordre religieux, en fidélité à l'idéologie du sacrifice typique de l'époque Zhou tardive. Il présente le $w u$ comme le chef d'une hiérarchie d'officiers, en harmonie avec la construction d'un ordre rituel qui se met en place à l'époque où le texte est écrit (Keightley, 1998, p. 821-824). Quant au Zhouli (Classique de Rites des Zhou, date de compilation incertaine, mais probablement à dater du tout début de l'ère chrétienne), il présente l'activité des $w u$ - masculins et féminins - en lien étroit avec les événements funestes : maladies contagieuses, sécheresses, inondations... Ils sont engagés et congédiés en fonction de l'efficacité de leurs performances. Ils sont dirigés par deux officiers qui ne semblent pas eux-mêmes être des $w u$ (Von Falkenhausen, 1995, p. 291-292). Le développement des activités thérapeutiques menées par des $w u$ est suggéré par d'autres textes. Pourtant, là encore, des différences entre le rôle du $w u$ et celui du chamane sibérien peuvent être notées : en Sibérie, comme c'est aussi le cas 
dans de nombreuses minorités ethniques de l'Ouest de la Chine, le chamane accomplit son œuvre thérapeutique par l'identification de l'esprit responsable de la maladie puis en l'apaisant et le chassant. Dans la Chine archaïque, le sacrifice semble destiné à identifier et apaiser l'esprit, et le $w u$ se contente de l'expulser. Plus généralement, plusieurs extraits du Liji (ouvrage rituel associé au Zhouli) et du Zuozhuan (ouvrage historique probablement très antérieur aux précédents) suggèrent que le rôle du $w u$ est d'abord d'éviter la propagation des malheurs. Sa présence ne semble donc guère auspicieuse. Dans la même ligne, la capacité du $w u$ d'interpréter les rêves semble avoir été considérée comme dangereuse. Plus globalement, comme l'attestent plusieurs auteurs (voir par exemple Xunzi, chap. XVII), les danses des femmes $w u$ étaient liées aux prières pour l'obtention de la pluie. Ces danses étaient donc marquées par de fortes connotations sexuelles. Les fêtes sexuelles associées à ces danses sont déjà l'occasion de désordre social (Wang, 2001). Mais le potentiel de désordre est redoublé par le possible déferlement de l'élément aquatique : pas assez d'eau est un malheur, trop d'eau aussi... Le pouvoir politique devait donc se mesurer avec prudence mais aussi, quand il le fallait, avec sévérité aux pouvoirs magiques des $w u$ (Boileau, 2002, p. 373-375).

En résumé, si l'image du $w u$ durant la dynastie Shang reste très confuse, les sources nous la restituent de façon plus précise pour la dynastie Zhou : le wu (mâle ou femelle) est un spécialiste des événements malheureux, considéré avec appréhension par la population comme par les pouvoirs politiques, un spécialiste dont l'action est politiquement encadrée, de la même façon que l'eau est contrôlée au travers d'ouvrages hydrauliques, afin que le corps social bénéficie de son pouvoir sans souffrir de son débordement. Son action se déroulait souvent dans des lieux sauvages, et impliquait sans doute un contact physique avec les morts. Enfin, les sources assez abondantes sur les $w u$ trouvées dans les sources Zhou de l'Est ont très peu en commun avec les rares indications des sources Shang, et l'on peut même se demander si le même terme ne s'est pas appliqué consécutivement à des réalités très différentes selon les époques (Keegan, 1979). La différence de nature entre le wu des Shang et celui des Zhou pourrait être paradoxalement prouvée par la survivance du système le plus ancien dans l'État de Chu, établi autour du Yangzi, durant la dynastie des Zhou : la religion de l'État de Chu aurait continué à être marquée par l'iconographie animale de la religion Shang, une insistance sur la chasse considérée comme activité rituelle, une grande importance donnée à la transe chamanique et à la capacité du médium de sortir de son corps pour effectuer un voyage au travers de l'espace et du temps (Major, 1999, p. 124). Cette survivance serait partiellement explicable par l'origine ethnique de la population dominante de Chu, originaire des steppes mongoliennes à la suite d'une migration étant intervenu à la fin du second millénaire av. J.-C. (Major, 1978, p. 231). Nous ne nous étendrons pas davantage ici sur ce cas controversé. Il montre en tout cas que la question de la transformation 
et de la survivance de la religion chinoise archaïque (que cette dernière puisse ou non être qualifiée de chamanique) exige que l'on vienne avec des réponses géographiques différenciées, ce que les découvertes archéologiques des dernières décennies nous permettent désormais de tenter avec plus de précision qu'auparavant (voir ainsi Von Falkenhausen, 2006, notamment chapitres I, IV et VII).

Comme la plupart des autres ouvrages chinois sur la question, le livre de Zhao Zhizhong ici recensé se garde d'une approche trop critique. Après avoir défini la notion de "sphère chamanique » et isolé plusieurs ensembles géographiques qui lui correspondraient, il inclut parmi elles le Nord-Est et le NordOuest chinois. Le recensement de certains des textes évoqués plus haut lui permet d'affirmer que « la Chine est le pays du monde qui a le plus anciennement mentionné et étudié le chamanisme » (Zhao, 2008, p. 5) - la revendication de la primauté chronologique constituant l'inévitable entrée en matière de la plupart des travaux en sciences humaines et sociales du monde chinois. Les considérations théoriques qui suivent sont tout aussi décevantes, mais le travail ethnographique réalisé dans les provinces du Nord-Est chinois livre une riche moisson de textes et de chants, rehaussée par un travail de transcription soigné. Ouvrage typique d'une littérature chinoise "à l'ancienne ", encore marquée par l'ethnographie soviétique, avec ses capacités de collationnement et ses évidentes limites. Le renouvellement théorique est encore peu perceptible. Fait partiellement exception un article de Meng Huiying (Meng, 2010) qui esquisse avec clarté les débats en cours en d'autres contextes culturels, notamment sur le rapport du chamane à la communauté dans laquelle il officie et sur les rapports entre expressions rituelles traditionnelles et reconstructions identitaires. Il reste encore à appliquer ces problématiques aux minorités ethniques du Nord-Est, Nord-Ouest et SudOuest chinois - une entreprise malaisée lorsque la plupart des ethnographes chinois préfèrent souligner la contribution des dites minorités à la culture nationale prise en sa globalité.

\section{De la Chine au monde austronésien}

Les différents groupes aborigènes taïwanais appartiennent tous à la famille linguistique austronésienne, et sont à l'origine de la grande expansion austronésienne qui a marqué l'histoire du Pacifique et de l'Asie du Sud-Est. Les premiers départs depuis Taiwan vers les Philippines semblent avoir eu lieu il y a environ quatre mille ans et se sont ensuite poursuivis, d'une part vers l'Indonésie jusqu'à Madagascar, de l'autre dans tout le Pacifique jusqu'à l'île de Pâques. D'un point de vue linguistique, on peut même dire que l'expansion austronésienne continue encore aujourd'hui (Lansing et al., 2011 ; Donohue \& Denham, 2010 ; Bellwood, 1978). L'observation d'une tradition chamanique propre aux aborigènes de Taiwan est donc particulièrement intéressante pour la compréhension de la culture et de la structure de pensée de la grande famille linguisticoculturelle austronésienne. 
Aujourd'hui, les rituels aborigènes encore observables sont essentiellement dirigés par des femmes. Mais cela peut correspondre à différents types d'évolution sociale. Dans le cas des Puyuma, au sud de la côte Pacifique, le passage tardif de chamanes hommes aux chamanes femmes est sans doute lié à la prédominance progressive de l'agriculture sur la chasse, notamment du fait de l'interdiction de la chasse qu'avait cherché à imposer l'administration japonaise. En réaction à ce changement, certains mythes tardifs témoignent alors du choix explicite fait par les esprits de personnes effectuant "les travaux des champs ", c'est-à-dire des femmes, ces mêmes esprits ancestraux élisant comme intermédiaire et fondateur du nouveau régime chamanique un "homme efféminé » (Cauquelin, 1991 et 2004). Le cas des Puyuma s'inscrit donc dans celui des sociétés où la féminisation de la fonction chamanique est liée à une profonde transformation sociale. Elle va de pair avec la modification des pratiques dès lors essentiellement thérapeutiques.

Dans le cas des Kavalan, aborigènes des plaines du Nord de la côte Pacifique, Liu Pi-chen observe une situation différente : le chamane est par principe une femme. C'est une situation fort rare dans les sociétés chamaniques, même dans les sociétés matrilinéaires (comme le sont les sociétés kavalan et amis). Ici, le pouvoir féminin est assuré, d'une part par un système matrilocal, d'autre part par le contrôle des rites chamaniques : aussi bien la matrilocalité que le bon déroulement des rites ont pour fonction symbolique d'assurer la transmission régulière de la vie, explique Liu Pi-chen. En contraste, les hommes kavalan sont organisés par classes d'âge : les classes d'âges forment la base des activités chasseresse et guerrière, qui sont des activités aléatoires par excellence (Liu Pi-chen, 2007). Chez les Kavalan, les mythes d'origine mettent en valeur le rôle enseignant d'une déesse qui apprit aux mtiu (groupe de femmes chamanes) l'art des rites chamaniques (kisaiz et pakelabi) pour éviter les calamités et guérir les maladies. Chez ces peuples riverains du Pacifique, certains de ces mythes d'origine mettent en valeur l'échange de dons nécessaire au bon déroulement de la pêche (qui jouerait alors un rôle similaire à celui observé pour la chasse dans les sociétés sibériennes). La société Amis (le plus grand des groupes aborigènes de Taiwan) connaît également des chamanes (sikawasay), majoritairement des femmes, mais aussi parfois des hommes. Dans les deux cas (kavalan et amis), il s'agit d'échanger des biens avec les esprits au cours d'un voyage symbolique dans l'au-delà afin d'obtenir les faveurs désirées. Le rite et les chants figurent à la fois ce voyage dans l'au-delà et l'histoire mythique du village ou de la tribu qui accomplit le rituel. Échangeant des biens avec les esprits, la communauté qui assiste au rite affirme ses relations de sang avec ces mêmes esprits et renforce en même temps son unité intérieure. Pour conclure et renouveler l'alliance tant de la communauté avec elle-même que de la communauté avec la surnature, le langage formalisé $\mathrm{du}$ rite et du chant prend la place du langage écrit, traditionnellement absent de ces sociétés (Liu Pi-chen, 2008 et 2011). La fonction chamanique des femmes dans ces deux sociétés apparaît donc comme un fait originaire, lié à la structure 
matrilocale et au mode de répartition du travail entre hommes et femmes. C'est plutôt l'introduction des religions catholique et protestante qui aurait diminué le rôle religieux et social de la femme au cours de la période récente (Liu Pi-chen, 2003 et 2007). Sur ce dernier point, il faut faire preuve de prudence : l'observation montre qu'à Taiwan les anciennes chamanesses fournissent une proportion importante des catéchistes des Églises chrétiennes, et qu'elles jouent souvent un rôle dirigeant dans ces Églises (Hu et al., 2008, p. 80-229). Et si la plupart des Églises protestantes (mais pas toutes) se sont montrées très hostiles envers le chamanisme, les prêtres catholiques ont nuancé leurs jugements en fonction des cas, et se sont montrés plus accommodants avec les années.

À Taiwan, comme en tant d'autres endroits, l'activité chamanique ne saurait s'appréhender comme simple "tradition " à préserver : en premier lieu, les formes constatées chez les différents groupes aborigènes ont été progressivement influencées par le contact avec d'autres groupes ethniques. Ainsi, pour les Puyuma, Josiane Coquelin reproduit l'illustration d'un carnet de notes d'un devin (benabulu) où figurent des observations en langue japonaise. Plus tard, les chamanes puyuma utiliseront pour leurs rites des transcriptions en langue chinoise (Cauquelin, 1991 et 2004). Par ailleurs, dans la période la plus récente, l'activité chamanique et rituelle est devenue partie d'un mouvement de recréation de l'identité ethnique et culturelle. Michael Rudolph a attiré l'attention sur ce qu'il appelle des « retraditionalizing rituals » (Rudolph, 2008, p. 15), lesquels, au travers d'un usage mouvant de l'histoire et de la tradition, influent sur la gestion des conflits de pouvoir à l'intérieur du village, les relations entre les ethnies aborigènes et le gouvernement central, ou d'autres situations à forte charge symbolique. Le rituel est la matrice de "sens émergents ", qui prennent naissance au travers de la subversion des symboles cosmologiques traditionnels (cf. Tambiah, 1979). Les modifications qui prennent place dans la célébration témoignent d'un travail de sens au travers duquel la communauté redéfinit la manière dont elle se comprend. Compte alors moins la symbolique apparente du rituel que l'effet de sens et de pouvoir associé à sa performance. Dans le contexte taïwanais, Rudolph illustre cette approche en rendant compte de la transformation des esprits-ancêtres (ancestor spirits) : traditionnellement " démons " qu'il faut exorciser et chasser, ces esprits-ancêtres deviennent par étapes les forces qui protègent et soutiennent la communauté aborigène dans son entreprise de renaissance culturelle et politique. Parallèlement, la célébration rituelle devient un enjeu politique, disputé par des forces rivales : des groupes aborigènes revendiquent leur passé de "chasseurs de têtes » pour donner plus d'éclat à leurs protestations contre les gouvernements successifs. L'émergence d'une classe d'intellectuels aborigènes contribue à la manipulation du capital symbolique que représentent les rituels traditionnels. Un autre point mérite attention : le renouveau et la transformation des pratiques rituelles ouvre un espace de " négociation » entre la foi chrétienne qui est désormais celle de la grande majorité des aborigènes taïwanais et un passé qu'ils se réapproprient en le réinventant. 
«Négociation » parfois malaisée : le degré d'adhésion des diverses communautés aborigènes à telle ou telle église chrétienne restreint leur liberté dans la réinvention rituelle (Qiu Yunfang, 2004). Difficulté redoublée par le fait que l'investissement stratégique dans les rituels s'est accompagné d'une insistance renouvelée sur leur caractère religieux, parfois illustrée par un sacrifice d'animal (Rudolph, 2008, p. 198-201). En d'autres termes, les enjeux de pouvoir liés à la redéfinition des rituels amènent leurs promoteurs à insister sur leur caractère "sacré ", dont témoignera leur mode de mise en spectacle.

Un cas limite est atteint avec la société thao, le plus petit groupe aborigène de Taiwan avec moins de sept cents membres, un groupe à peu près complètement hanisé et où les épouses proviennent souvent des ethnies voisines, bunun notamment. Mitsuda Yayoi a étudié la relation entre leur stratégie de survie culturelle et le maintien de rites chamaniques (Mitsuda, 2010 et 2011). Les chefs masculins des sept clans thao se partagent la célébration de quatre rituels saisonniers, et gardent une certaine liberté dans les paroles prononcées à ces occasions. Cependant, l'activité religieuse principale est réservée aux cinq femmes chamanes (xianshengma dans l'appellation familière chinoise ordinairement utilisée) qui subsistent. Ces dernières, même quand elles n'appartiennent pas originairement à l'ethnie thao mémorisent strictement la mélodie et les paroles des rituels traditionnels, et les chantent sans en changer un mot. C'est en fait la seule occasion où la langue thao est encore employée. Ces rituels se tiennent en face des paniers de rotin et d'osier (ulalaluan), emplis d'étoffes ou autres objets appartenant aux générations passées, et dont chacun symbolise une maisonnée : fin 2009, on comptait cinquante-quatre ulalaluan, chiffre correspondant au nombre de maisonnées appartenant à l'ethnie Thao (Mitsuda, 2011, p. 132). Les rituels qui sont célébrés lors de déménagement, mariage ou funérailles s'effectuent devant ces paniers, réceptacles d'objets de toute nature. L'on a assisté à une évolution analogique de la fonction chamanique : les femmes chamanes sont comme ces " paniers » où sont gardés les objets (ici, les textes rituels) qui fondent encore la relation avec les ancêtres, garante de la survie précaire de l'ethnie.

Dans ses contributions sur les chamanesses de l'ethnie Paiwan (Hu, 2010 et 2011), Hu Tai-li a pareillement noté le caractère solidement fixé des récitations rituelles. Les chamanesses ne sont pas autorisées à changer les textes anciens, et sont considérées comme des médiums passifs des esprits ancestraux. Peutêtre faut-il rapprocher ce phénomène des caractéristiques de l'échange entre les humains et la surnature chez les Paiwan : l'obtention des animaux chassés ne vient pas d'un processus d'alliance matrimoniale, mais de l'échange entre porc sauvage (chassé) et porc domestiqué : la façon d'obtenir les animaux sauvages désirés est de tuer un porc domestique et de le préparer soigneusement, comme une offrande qui sera faite durant le rituel chamanique, espérant que les esprits ancestraux accepteront le porc domestiqué et accorderont en échange les animaux sauvages (Hu, 2011, p. 18-20). La passivité des chamanesses paiwan se 
traduit par la rigidité manifestée dans la manière d'interpréter les chants, selon un processus que $\mathrm{Hu}$ Tai-li, suivant Joël Kuipers, nomme entextualization (Hu, 2011, p. 25-27 ; Kuipers, 1990) : contrairement à la contextualisation, qui adapte la performance dramatique aux circonstances qui la justifient, l'entextualization codifie et rigidifie de plus en plus la récitation et le chant, quelles que soient les circonstances de la performance. Comme chez les Thao, et même si les raisons en sont quelque peu différentes, les chamanesses sont d'abord et avant tout les gardiennes d'un ordre menacé et les dépositaires d'une mémoire fragile. L'entextualisation manifeste l'évolution de leur rôle social en direction de la fonction de dépositaire.

\section{L'aire chamanique d'origine}

Comme nous l'avons vu, une définition du chamanisme, fondée essentiellement sur des critères historiques et linguistiques, voit dans l'aire sibérienne le monde chamanique par excellence. Même cette approche élargit pourtant le plus souvent l'application du terme aux régions limitrophes : Mongolie, Mandchourie et Corée principalement (les cas du Tibet, du Népal, et, plus largement, de l'aire himalayenne, posent un problème particulier que j'aborderai en fin de cette partie). Rappelons et précisons ce qui caractérise le chamanisme dans son aire géographique d'origine :

«Il est le système symbolique conçu pour instaurer et faire fonctionner l'échange avec les esprits des animaux sauvages. Telle est l'essence de la fonction chamanique : établir un contrat qui légitimise par avance de prendre du gibier et comporte une promesse de contrepartie. Ce contrat est défini, en Sibérie, sous sa forme la plus contraignante, celle de l'alliance matrimoniale, dont la logique implique un enchaînement sans fin : tout homme qui prend une femme en mariage devra un jour donner sa fille à un mari, et ainsi de suite. Posée comme cadre institutionnel de l'échange, l'alliance en garantit la perpétuelle reconduction. L'un et l'autre sont orientés, faisant du partenaire humain un preneur de femme et de gibier. L'alliance fixe à la communauté humaine comme telle, et plus concrètement au chamane qu'elle charge de la représenter, la position du mari. [...] La fonction chamanique s'exerce dans le cadre de rituels collectifs périodiques, dont les noms reflètent les retombées positives escomptées. » (Hamayon, 2006, p. 23-24).

Ce n'est pas sur la mouture classique du chamanisme dans ces régions que nous nous attarderons, mais plutôt sur les études décrivant la synthèse entre chamanisme et modernité dans ces mêmes régions, en même temps que le décalage entre l'idéal type et la réalité contemporaine.

La disparition progressive du chamanisme en Mongolie extérieure est un phénomène largement documenté. La conversion d'Altan Khan au bouddhisme Gelugpa en 1578 avait déjà entraîné la destruction de nombreuses figurines chamaniques, remplacées par des icônes bouddhiques, et les éleveurs de chevaux réfractaires à la conversion s'étaient vus confisquer leurs chevaux (Bawden, 1968, p. 26-37). Avant même cette période, plusieurs grands rituels chamaniques 
(par exemple pour éviter les calamités en cas de gros orage) avaient déjà été très directement influencés par des rituels en provenance du bouddhisme tibétain (un exemple de survivance contemporaine de ces rituels syncrétistes est donné par Birtalan, 2001). Au fur et à mesure du temps, la pratique chamanique est de plus en plus marginalisée, confinée à la taïga septentrionale. Par ailleurs, et surtout, si la politique religieuse du gouvernement mongol des années 1920 à la fin des années 1980 n'a pu déraciner le bouddhisme, elle a achevé de porter un coup fatal aux pratiques chamaniques. Certes, on peut observer à Ulaanbaatar un certain renouveau néo-chamaniste (Merli, 2004), mais il est d'ampleur limitée, et sans influence sur le reste du pays. Cependant, les études ethnographiques, et notamment celles portant sur les récits populaires, discernent sans trop de peine des éléments chamaniques toujours vivants. La croyance selon laquelle seules certaines personnes sont capables à la fois de voir les " fantômes » ou les « esprits » et d'entretenir une relation, un dialogue avec eux reste enraciné en plusieurs régions (Humphrey \& Onon, 1996). Les raisons de cette constance culturelle chamanique, alors même que les structures religieuses correspondantes ont disparu, sont liées à une quête identitaire nationale, mais pas seulement. L'analyse des récits populaires montre que certaines régions du territoire mongol « portent le deuil " de la disparition des chamanes : les histoires de fantômes qu'on y raconte sont celles de personnes (y compris de lamas bouddhistes) qui « voient » les esprits au cours d'un déplacement nocturne, mais qui ne peuvent pas interagir avec eux, et qui doivent donc se contenter, une fois de retour chez eux, de tenir un rituel pour éviter que pareille rencontre leur soit néfaste (Deleplace, 2009, p. 281-282). Voir, savoir, pouvoir (voir, les esprits, savoir qui ils sont, pouvoir les contrôler)... Ces opérations qui, traditionnellement, définissaient la compétence du chamane semblent être désormais dispersées entre plusieurs agents religieux. Pour dire les choses autrement, ce qu'on observe dans la Mongolie actuelle, c'est « la réinvention d'une cosmologie chamanique sans chamane " (Delaplace, 2009, p. 282). Mais des survivances plus directes peuvent être relevées: en témoignent les contes qui décrivent les esprits de certains morts sous l'aspect de petits bonshommes malfaisants qu'il s'agit de capturer et de neutraliser pour éviter que le malheur s'abatte sur la famille. Ces contes contemporains rappellent ce qui fut l'une des tâches principales des chamanes mongols : comme certaines maladies étaient supposées être causées par l'action néfaste d'un esprit de mort frustré, cherchant à se venger de son malheur sur les humains, le chamane mongol devait identifier l'esprit fauteur de trouble et l'introduire dans une figurine qui serait nourrie et choyée par la famille. Il s'agissait donc de guérir le malade en faisant de l'esprit pathogène un ancêtre protecteur. Pour ce genre d'entreprise, le chamane s'appuyait sur ses propres esprits qu'il avait incorporés au fur et à mesure de sa pratique thérapeutique (Delaplace, 2009, p. 284-287).

Dans la même ligne, Daniel Kister commente un rituel bouriate contemporain tenu près du lac Baikal d'une façon qui entend tout à la fois marquer une distance 
par rapport au récit traditionnel sur la cérémonie chamanique tout en préservant la sensibilité littéraire et artistique qui en a marqué le style narratif : «Les organisateurs de ces rituels sont des "artistes rituels" et non pas des chamanes sibériens traditionnels. Les participants ne constituent pas non plus une communauté chamanique classique. Ils constituent un groupement temporaire sensible à l'appel esthétique de la nature, pas un groupe établi qui se tournerait vers les dieux de la nature. Pour eux, il n'y a pas de manifestation concrète des forces surnaturelles. Quoique le but explicite du rituel soit de rendre grâce au dieu de l'eau, le processus réel est dirigé vers "le développement personnel" des participants, comme le fait est typique dans un rite néo-chamanique. [...] On ne trouve pas là des images originales ou des codes symboliques complexes qui créeraient une façon de se situer dans le monde ayant le pouvoir d'enrichir durablement l'expérience communautaire de la réalité. »(Kister, 2010, p. 251-253).

Les rites chamaniques mandchous sont également en voie de disparition presque totale, mais ils se situent à l'intersection de ceux du monde mongol et sibérien, que nous venons d'évoquer, et de ceux du monde coréen, dont nous parlerons immédiatement après. Historiquement, on sait l'importance que jouaient les rites chamaniques pour la dynastie mandchoue : ils étaient régulièrement célébrés au cœur même de la Cité interdite. Le Rituel impérial mandchou (Hesei toktobuha Manjusai wecere metere kooli bithe) de 1747 les décrit avec force détails (Liu Housheng, 1993 ; Jiang, 1995 ; Bai, 1996). Les relations entre ces grands rites impériaux et les rites populaires de taille modeste que l'on peut encore parfois observer dans la province de Jilin peuvent paraître lointaines. Mais à l'occasion des connexions directes peuvent être trouvées : c'est ainsi que Giovanni Stary a pu connecter le verbe mandchou tuimbubi (habituellement traduit par "prier dans le noir, après avoir éteint les lanternes ») avec les sacrifices et les invocations de rite populaire encore observable au début des années 1980 dans le Jilin (Stary, 2007).

On observe des différences significatives entre rituels chamaniques mandchous et coréens. Tout d'abord, dans le monde coréen les chamanes sont surtout des femmes, tandis qu'ils sont masculins en Mandchourie. Les rites mandchous contemporains sont aussi plus conventionnels que les rituels coréens, très axés sur les problèmes immédiats (conflit familial larvé, deuil douloureux, maladies à répétition, malchance) rencontrés par la famille qui organise le rite. Ils sont surtout beaucoup moins fréquents, et moins chargés d'émotion. Mais dans les deux cas subsiste la croyance que le chamane a rencontré dans sa vie l'intervention d'une force surnaturelle et a été transformé par cette force (Kister, 2010, p. 69-70). Nous parlerons des rites chamaniques coréens dans la partie suivante. Notons déjà que le terme mudang (terme typiquement coréen et non pas emprunté au chinois) apparaît pour la première fois dans un récit du XII siècle, et que la parole prononcée par ce devin (masculin ou féminin, on ne le sait) le fait mettre à mort par le roi. Même si les différences régionales sont nombreuses, 
la mudang (ou parfois un chamane masculin) intervient dans un rituel (kut ou gut selon la romanisation utilisée) où elle personnifie les dieux ou les âmes des morts qui interviennent à l'occasion du problème familial pour laquelle le rituel est organisé. La qualité de spontanéité du rituel, typique d'un « théâtre populaire ", contraste avec l'orthodoxie confucéenne qui marque la société coréenne. De même, le fait que les intervenants soient essentiellement des femmes tranche avec la dominance masculine qui marque cette même société. Le rituel chamanique est donc un « jeu sérieux » : " les dieux sont presque humains, les ancêtres presque divins, et les dieux, les ancêtres et les hommes deviennent Un dans les hauts et les bas chaotiques de la vie»(Kister, 2010, p. 221).

On notera également les relations riches et complexes qui ont été nouées entre le chamanisme et les autres religions actives en Corée. Ainsi, la théologie coréenne de la libération a comparé souvent Jésus à un chamane, exprimant et guérissant le ressentiment et la douleur ( han) exprimés par le petit peuple. Le han est un terme qui réfère à une structure sociopsychique complexe. Il comporte frustration, regret et ressentiment influençant les relations entre les vivants ou parfois entre les vivants et les morts (Kister, 2010, p. 178). La proclamation du Royaume de Dieu est elle-même comparée à un rituel chamanique, et le théologien fait alors réciproquement de la tradition chamanique une voie d'accès à la connaissance de Dieu (Choi Ki-bok, 1995 ; Kim, 1989). Daniel Kister écrit : "La communauté qui célèbre le kut est une communauté de personnes qui cherchent à être activement libérées de l'affliction, et qui est ouverte à une présence divine au travail dans la nature, la maisonnée, dans les zones de crise que sont naissance, maladie, mort, dans la vie de la chamane, et dans le jeu et la prière de leur propre communauté. C'est une communauté de personnes qui célèbrent leur vie ensemble dans des symboles transformants, parfois ordinaires, parfois sacramentaux, parfois stupéfiants, parfois beaux, et toujours finalement dramatiques. »(Kister, 1997, p. 160-161).

Nous avons déjà évoqué la continuité entre sociétés mongole et tibétaine - et, plus largement, himalayenne. À l'est de la zone culturelle tibétaine, dans la région du Kham si l'orthodoxie Gelugpa est établie, la vitalité exhibée (notamment au sud-est) par d'autres écoles tantriques est un signe d'une identité spécifique. La persistance de la tradition Bön (populaire et souvent cachée) témoigne aussi de contacts continus avec les religions proto-chamaniques des minorités avoisinantes. «La religion Bön [...] s'est maintenue au milieu du lamaïsme dont elle a reçu nombre de ses idées et de ses doctrines, sans qu'il fût pourtant en mesure de l'étouffer entièrement. " (Tucci \& Herssig, 1973, p. 271). La religion Bön s'est donc maintenue parce qu'elle a évolué. Loin d'avoir subi un déclin continu, elle a connu, au cours de sa longue histoire, plusieurs renouveaux et reformulations. Parallèlement, le lamaïsme s'est acculturé en reprenant à son compte de nombreux rituels ancrés dans la tradition Bön. Geoffrey Samuel a longuement développé la thèse selon laquelle le bouddhisme tibétain était constitué de différentes synthèses entre une tendance «cléricale » et une tendance 
"chamanique "- on peut le définir en fin de compte, affirme-t-il, comme un "bouddhisme chamanique " (Samuel, 1993). Cette thèse a cependant été critiquée par plusieurs chercheurs : elle a trop tendance à reprendre comme tel le discours bouddhiste qui, historiquement, s'est toujours défini comme religion "civilisée » en contraste avec les religions locales «barbares » des territoires qu'il rencontre (Obadia, 2006).

Au Népal, le chamanisme n'a fait l'objet d'études que très tardivement, en raison de la prédominance des deux grandes traditions qui se partagent le pays, à savoir l'hindouisme ( $85 \%$ environ de la population) et le bouddhisme (7\% environ de la population). Pourtant, dans l'ensemble du Népal, les chamanes (dhami-jhankris) occupent une place essentielle dans la vie religieuse et culturelle, en faisant coexister le chamanisme avec d'autres religions. Certains groupes ethniques apparaissent comme de véritables "sociétés chamaniques » (de Sales, 1991), d'autres n'ont adopté du chamanisme que certaines pratiques, qui demeurent marginales par rapport à des systèmes de croyances comme l'hindouisme. Par ailleurs, les rapports entre chamanisme et bouddhisme sont, dans ces régions de l'Himalaya, bien plus souvent caractérisés par la conflictualité (même larvée) que par la cordialité. Ainsi, les Sherpas ont longuement pratiqué un bouddhisme " populaire ", avec des lamas mariés vivant au cœur des villages. La construction des grands monastères, au début du XIX ${ }^{\mathrm{e}}$ siècle, consécutive de changements économiques, a conduit à l'alignement de ces formes populaires sur un monachisme orthodoxe, bien moins enclin à tolérer la présence des chamanes. Le déclin du chamanisme a ainsi été formulé comme relevant de la pression exercée par le bouddhisme pour absorber son rival local (Ortner, 1998). Selon la théorie d'Ortner, tout se serait joué dans la conquête politique de la société sherpa par le bouddhisme, une tradition plus tardive, organisée autour d'un puissant appareil monastique, fondée autour d'un corpus doctrinal structuré, et donc une religion plus «moderne » que le chamanisme, lequel repose sur le charisme de ses spécialistes et leur inventivité dans le domaine magique. En l'absence de données historiques fiables, il s'avère difficile d'affirmer avec cet auteur que le phénomène d'institutionnalisation du bouddhisme est directement à l'origine des vicissitudes du chamanisme, ou des tensions entre les traditions religieuses locales. Il n'en demeure pas moins que les observateurs de la vie religieuse locale ont relevé, avec régularité, l'expression de ces antagonismes, qui prennent la forme, sur le versant bouddhiste, de campagnes contre des chamanes supposés verser dans des pratiques archä̈ques - contraires à la vision d'un bouddhisme porteur de valeurs plus évoluées, plus respectueuses de l'écologie locale, et œuvrant pour le bien de la collectivité dans son ensemble. Aux accusations d' "infériorité » et de "barbarie » qui leur sont adressées, les chamanes répliquent par la condamnation d'un monachisme bouddhiste irrespectueux des forces invisibles qui se rebellent en attaquant les hommes et les récoltes (Gibson, 1997 ; Ortner, 1998 ; Obadia, 2006). 
Enfin, nous ne ferons ici que mentionner les chamanismes des minorités ethniques du sud-ouest de la Chine. Il faut les considérer comme une continuation des chamanismes du nord-ouest de la Chine et du monde himalayen. Les Yi de Liangshan notamment connaissent un système comportant deux intermédiaires religieux, l'un sachant lire et dont la légitimité est confirmée par son appartenance à une lignée patriarcale (le bimo), l'autre identifié comme chamane par des esprits tutélaires lors de crises psychiques intervenant à certains moments de sa vie, et ne sachant généralement pas lire (le ou la suni, un type religieux dont les études de terrain montrent qu'il est sans doute actuellement en voie de disparition). Le bimo et le suni peuvent l'un et l'autre intervenir pour la guérison de maladies, mais seuls les bimo peuvent présider à la conduite des âmes des défunts vers le monde des ancêtres - une affaire complexe, qui peut s'étendre sur une période de vingt ou trente ans. Traditionnellement, il semble que le bimo présidait aussi aux grands rituels de chasse ou de guerre. Les deux modèles correspondent bien au prototype chamanique tel que décrit pour les sociétés présentées à l'instant. Le chamane reste maître de ses esprits et conclut avec eux une alliance qui le lie à eux pour la durée de sa vie. On notera la remarquable persistance de ce système au cours des âges, les Yi de Liangshan ayant résisté à l'influence religieuse de leurs puissants voisins Han et Tibétains. Jusqu'à un certain point, la société Yi de Liangshan est donc encore une société de type chamanique, quelles que soient les transformations qui l'affectent (Ma Changshou, 2006 ; Bamo,1994 ; Ma Erzi, 2009 et 2010).

La culture religieuse traditionnelle des minorités tibéto-birmanes du Yunnan a été bien plus nettement affaiblie que celle des Yi de Liangshan, tant du fait de leur longue proximité avec les Han que par la christianisation de nombreux villages à partir de la fin du XIX ${ }^{\mathrm{e}}$ siècle. Certains ouvrages retracent néanmoins la survie de rituels saisonniers, sans pourtant caractériser vraiment ce qu'ils gardent encore de "chamaniques" (Nevot, 2008). Pour sa part, Éric Mueggler a montré comment l'héritage religieux local de certaines populations du nord du Yunnan a pu les aider, après la Révolution culturelle, à articuler leur mémoire, leur identité et les contradictions qu'il rencontrent dans une langue symbolique qui a organisé le territoire qu'ils habitent sous la métaphore d'un "corps souffrant » que les rites cherchent à guérir (Mueggler, 2001). En d'autres termes, la recherche à poursuivre sur les minorités ethniques du Sud-Ouest de la Chine devra rendre compte tout à la fois de leur héritage religieux, de leur identité sociale, et des rituels modernisés qu'ils mettent en place lorsqu'ils articulent cet héritage et cette identité.

\section{Entre théâtre et thérapie}

L'un des premiers ethnographes décrivant en profondeur le chamanisme toungouse notait déjà : "L'acteur est la communauté elle-même. Le chamane ne peut être considéré que comme un organe spécifique de la communauté. » 
(Shirokogoroff, 1935, p. 335). Le chamane se doit d'avoir avec lui une communauté qui croit en ses pouvoirs et en ses connaissances, et "c'est cette qualité qui sépare le chamane d'un voyant ou d'un extatique individuel » (Gibson, 1997, p. 44). En même temps que le caractère communautaire du chamanisme a été réaffirmé par les travaux poursuivis durant ces deux dernières décennies, les fonctions thérapeutiques du chamane, valorisées par Eliade et l'école américaine constituée dans sa mouvance, ont été minimisées au profit d'un nouvel accent sur les fonctions typiques des sociétés chamaniques traditionnelles : identification des proies pour la chasse, recherche des objets perdus, rituels destinés à assurer la survie physique et symbolique de la communauté.

Pourtant, les anthropologues ont bien dû continuer à se confronter au rapport entre chamanisme et thérapie - thérapie individuelle comme thérapie collective et l'insistance sur la fonction thérapeutique du chamanisme ne semble pas être en contradiction avec la reconnaissance du fait que c'est la communauté comme telle qui confère son rôle et sa légitimité au chamane. Simplement, il faut aussi tirer les conséquences du caractère social tant du don thérapeutique du chamane que de la technique dont il fait usage. Le chamane, dans les descriptions offertes par Eliade, mais aussi par des enquêtes plus récentes, est un homme ou une femme qui a expérimenté des troubles mentaux, soit à l'adolescence soit plus tard, troubles que l'acceptation de sa condition de chamane a aidé à tenir sous contrôle ou même à guérir (Eliade, 1951, p. 36-43; Devereux, 1970, p. 15). L'initiation chamanique, qui joue un rôle cathartique pour le chamane ou la chamanesse intronisé dans sa nouvelle identité (Kister 2010, p. 3-28) va souvent de pair avec l'infliction d'une peine physique (passage par le feu, marche sur des couteaux) qui, en permettant de dompter la peur, provoque le sentiment d'expérimenter une nouvelle naissance (Hoppal, 1992, p. 154-155). Le rituel initiatique nourrit la croyance du chamane - et la croyance du chamane est la condition même de l'efficacité du rituel (Kister, 2010, p. 95). Ce processus en boucle ne fonctionne pas à tous les coups, et, surtout dans les sociétés contemporaines, l'aspirant chamane est confronté à des émotions et des influences contradictoires : nous avons un récit visuellement documenté de l'échec de l'initiation d'une jeune femme au rôle de chamanesse coréenne ; dans ce cas précis, le pouvoir de transformation du rite ne pouvait pas jouer sur cette personne, restée emprisonnée dans son histoire personnelle et familiale (Kendall, 1991 et 1996). Cela dit, le caractère héréditaire de nombreuses lignées de chamanes et la diversité des trajectoires individuelles d'accès à la fonction interdisent d'ériger ce modèle du "fou guéri » en modèle unique. De nombreux auteurs ont d'ailleurs souligné la santé mentale que doit posséder le chamane pour porter ainsi les maux psychiques d'une multitude d'individus, et ont critiqué la conception psychopathologique du chamanisme et de la possession (Hamayon, 1995 ; Mitrani, 1982 ; Noll, 1982 ; Perrin, 1992 ; Siikala, 1978). 
Le questionnement sur le «sens du délire chamanique » ouvre celui sur la variété des «modèles symboliques " par lesquels il s'exprime : un rituel de divination ou un rituel chamanique se présente à la fois comme un enchaînement automatique de séquences et le lieu où se produisent des "trouvailles » dramatiques et symboliques plus ou moins nombreuses, inspirées par la situation qui a justifié la tenue du rite. Commentant la structure dramatique des rituels chamaniques coréens, Daniel Kister observe : «Un rituel chamanique a tendance à être fluide, flexible et "post-moderne" dans sa présentation. En contraste d'avec les drames littéraires, le texte oral change dans une certaine mesure à chaque séance. Néanmoins, en tant qu'œuvres créatrices qui expriment une manière fortement affirmée de se situer dans le monde, certains rituels coréens ( $g u t$ ) sont d'un niveau équivalent à celui des plus grands drames littéraires. "(Kister, 2010, p. 295). Plutôt que d'évaluer les troubles psychologiques ou la santé mentale initiale du chamane, il vaut mieux prêter attention à ses capacités de dramaturge et créateur, à la fois capable de respecter des formes fixes et de se trouver inspiré par les attentes et la participation du public (Kirby, 1975 ; Kister, 2010).

Il s'avère difficile d'aborder de front la question qui découle pourtant nécessairement des considérations précédentes : quelles sont donc la nature et la portée de l'efficacité thérapeutique associées aux pratiques chamaniques ? Un exemple caractéristique d'approche prudente et sceptique est fourni par un article sur les tang-kî taïwanais (Kleinman et Sung, 1979). L'observation d'un grand nombre de cas les amène à conclure que l'efficacité de la démarche thérapeutique ne concerne qu'un nombre limité de désordres psychologiques mineurs. Ils reconnaissent en même temps que la pratique des tang- $k \hat{\imath}$, en liant la perte de sens symbolique aux affections somatiques remplit un vide dans les soins que la médecine dispensée en hôpital est en mesure d'offrir (Kleinman et Sung, 1979, p. 22). L'approche de cet article exemplifie ce qu'on pourrait appeler le "modèle fonctionnel » du chamanisme et de la voyance : il existerait une fonction sociale du voyant, aux expressions variables selon les contextes, qui le situe à mi-chemin du médecin et du prêtre ou, plus exactement, qui comble l'intervalle entre les deux. Or, dans les sociétés contemporaines, la séparation grandissante entre les deux fonctions ouvre un espace où prolifèrent les «bricolages symboliques ». Un tel modèle ouvre des pistes intéressantes. Mais plus le schème d'explication se voudra fonctionnel, plus il tendra à privilégier la catégorie de simulation : plutôt qu'un mensonge conscient, l'analyste lira dans le comportement du chamane une attitude qui internalise la requête à lui adresser. Ici, véritablement, "la fonction crée l'organe »: la fonction symbolique crée cet "organe » qu'est le chamane : l'homme apte à "voir » dans le corps de son vis-à-vis le cours de son histoire et les voies de sa guérison. Geoffrey Samuel a proposé de dépasser un tel modèle, et a mis en question la distinction corps-esprit qu'une telle approche symbolique de la guérison chamanique implique. Pour lui, le langage rituel des esprits et de la possession joue indissociablement sur le mental et sur le physique, et la littéralité de ce langage doit donc être prise au sérieux : "I suggest that [...] we can 
treat the spirits as having a more focused and specific action than that of a generalized placebo, and as operating at both psychological and physiological levels, which are ultimately not very distinct from each other. To do this, however, we need to take the language of spirits, of magic, sorcery and ritual healing more seriously; not in terms of occult forces, but as providing tokens and images for operating on the structure of human life at all levels, within a framework which does not dichotomize between mind and body. " (Samuel, 2010, p. 16).

Les études de littérature comparée ont donc aidé au renouvellement de la compréhension de la «cure chamanique ». Étudiant la dramaturgie de séances chamaniques en Corée, en Mongolie, en Sibérie et en Chine, Daniel Kister a mis en lumière la relation entre l'efficacité du rite sur la santé psychique individuelle et collective d'une part, la qualité de la représentation théâtrale offerte de l'autre. Non pas qu'un rite "compliqué » soit forcément plus efficace. Comme en matière dramatique, c'est parfois la simplicité, l'unité de temps et d'action, qui assure l'efficacité cathartique du rituel. Kister montre notamment la parenté de certains rituels avec les techniques mises en place dans le théâtre de l'absurde (Ionesco) et le théâtre de la cruauté (Artaud) [Kister, 2010, p. 261-285].

Des intuitions semblables étaient déjà en œuvre dans les approches qui comparaient la séance chamanique à la cure psychanalytique, soit jungienne, soit freudienne. Lévi-Strauss avait déjà fait le rapprochement entre le chamane et le psychanalyste dans un article célèbre (Lévi-Strauss, 1949). Notamment dans le cas coréen, où la cure chamanique est explicitement tournée vers les sentiments négatifs accumulés au long de l'histoire personnelle et familiale, les comparaisons entre chamanisme et psychanalyse se font désormais beaucoup plus précises. Les différences de technique qui peuvent apparaître sont souvent liées au contexte culturel et beaucoup plus centrées sur le poids des obligations familiales dans le contexte coréen, ce qui explique le caractère de "thérapie de groupe " que revêt une session chamanique, session où les intermèdes comiques, la nourriture prise en commun et la mise en scène (costumes, danses) mêlent aussi le tragique au ludique dans une fusion qui est celle-là même de la vie quotidienne - une fusion qui concourt sans doute à l'efficacité du drame thérapeutique ainsi joué (Kim Hogarth, 2010).

Enfin, ces dernières années, la compréhension de l'activité thérapeutique du chamane s'est élargie et a souvent été exprimée par le terme de holistic healing. L'attention portée sur ce point vient de l'état social et culturel des communautés aborigènes des divers continents, communautés souvent marginalisées et fragilisées : la réappropriation des rituels chamaniques qui régissaient leur autocompréhension comme leur cosmogonie peut contribuer à une reconstruction communautaire. Brian McCoy prend l'exemple des aborigènes Punu du désert occidental du continent australien et de leurs guérisseurs (maparm) : leur pratique livre une compréhension de la «bonne santé » qui ne sépare pas les aspects corporels, sociaux et spirituels. La "santé » implique tout à la fois l'état de la 
famille, celui du pays environnant, et aussi la relation entretenue avec le passé ancestral et cosmique. L'importance attachée à l'état du pays d'alentour - les vents, les forces cosmiques éventuellement manipulées par des personnes mal intentionnées - est un aspect particulièrement important de la vision du monde des maparm et de leur pratique thérapeutique. McCoy note enfin le désir des aborigènes Punu de voir les pratiques et croyances de leurs guérisseurs, des cliniques où ils se rendent et des Églises chrétiennes auxquelles la plupart d'entre eux désormais adhèrent se fondre en un tout, de façon à unifier les forces sociales et spirituelles qui conditionnent la survie de leur peuple (McCoy, 2008). Deux études du chamanisme encore pratiqué sur l'île de Bornéo entendent élaborer la contribution qu'apportent les rituels tant à la survivance d'un système local de connaissance qu'à la santé globale de la communauté (Lasimbang, 2008 ; Fung, 2008). Le thème traditionnel des pouvoirs thérapeutiques du chamane a donc profondément évolué dans sa formulation : désormais il s'articule étroitement, d'une part avec les recherches épistémologiques sur les modes de « connaissance locale " (local knowledge), d'autre part avec la question globale de la survie de populations aborigènes un peu partout menacées dans leur existence, leur estime de soi et leur identité culturelle.

La plasticité des usages du terme « chamanisme » dans les ouvrages et articles ici recensés va de pair avec un questionnement sous-jacent, différent de celui qui a marqué tant l'époque ouverte par Eliade que le renouvellement de la discipline au cours des années 1980 et 1990 - un questionnement que j'exprimerais comme suit : comment les catégories déployées par les fluides systèmes "chamaniques » étudiés nous aident-elles à appréhender la modélisation des savoirs propres à une société donnée ? Ainsi, Veikko Anttonen revisite-t-il la catégorie de "sacré » ou de «surnature » : alors que les auteurs classiques, à commencer par Eliade, considéraient le sacré ou la surnature comme un "donné » préalable à l'activité du chamane, Anttonen insiste sur le fait qu'il faut d'abord voir comment le chamane et la société dont il fait partie visualisent le "sacré " par exemple à partir de noms de lieux ou de pouvoirs spéciaux dévolus à certains lieux tels que des lacs ou des collines élevées qui marquent la topologie du territoire. La séparation entre nature et surnature n'est pas intangible, elle fait partie d'un système de connaissance, et donc d'une façon plus générale de délimiter des frontières entre groupes et territoires (Anttonen, 2007; Siikala et Ulyashev, 2003). Allant plus loin, certains auteurs se demandent si l'activité chamanique ne peut pas nous aider à mieux comprendre l'activité cognitive humaine dans la manière dont sont traités différents types d'informations (Frecksa et Luna, 2007). Les mécanismes rituels utilisés pour obtenir la guérison d'un individu et ceux employés pour accéder à un ordre de connaissance supra-logique sont alors à comprendre comme un tout unifié. Enfin, dans la même ligne, la philosophie de la religion en ses développements récents se demande comment les formes religieuses associées au chamanisme peuvent nous aider à bouleverser les catégories 
de la discipline dans ses formulations occidentales (Sharma, 2006). Les formes religieuses associées au chamanisme permettent notamment de donner plus d'importance au caractère « spatial » des croyances et pratiques religieuses, alors que la tradition occidentale insiste plutôt sur leur caractère "temporel ": les noms de lieux, les représentations spatiales de la surnature, l'organisation concrète des espaces rituels fournissent des exemples de la manière dont le chamanisme et d'autres expressions des religions dites "premières " (primal religions) construisent leur espace-temps. Une fois encore, bien d'autres pistes de recherche pourraient être évoquées. Mais celles qui l'ont été dans cette revue critique montrent déjà la fécondité maintenue des études sur le chamanisme. Devenues un carrefour interdisciplinaire, ces études ont permis un dialogue entre historiens de la religion, anthropologues, psychologues et psychiatres, musicologues, linguistes ou philosophes... Le concept de «chamanisme » ne possède plus la belle cohérence qu'on lui accordait. Il a été critiqué, déconstruit, parfois reconstruit selon des moutures mutuellement inconciliables... Mais il garde une étrange force subversive : celle de questionner les frontières et les méthodes des disciplines académiques qui font appel à lui.

\author{
Benoît VERMANDER \\ Université Fudan, Shanghai \\ benoit.vermander@jesuites.com
}

\title{
Bibliographie
}

AntTonen Veikko, 2007, «Transcending Bodily and Territorial Boundaries. Reconceptualizing Shamanism as a Form of Religion ", Shaman, 2 (1-2), p. 5-21.

BAI Hongxi, 1996, "Qinggong tangzi jisi yanjiu » (Rites shamaniques dans le Palais impérial durant la dynastie mandchoue), Мinzu yanjiu, 1996, 4, p. 78-83.

BAmo Ayi, 1994, Yizu zuling xinyang yanjiu (Recherches sur les croyances yi quant aux esprits ancestraux), Chengdu, Presses des minorités ethniques du Sichuan.

BAwden Charles R., 1968, The Modern History of Mongolia, London, Wensfeld and Nicolson.

Bellwood Peter, 1978, Man's Conquest of the Pacific: The Prehistory of Southeast Asia and Oceania, Auckland, Collins.

Birtalan Agnes, 2001, "The Tibetan Weather-Magic Ritual of a Mongolian Shaman ", Shaman, IX (2), p. 119-142.

BoIleau Gilles, 2002, "Wu and Shaman ", Bulletin of the School of Oriental and African Studies, 65 (2), p. 350-378.

Boyer-Araujo Véronique, 1993, Femmes et cultes de possession au Brésil, Paris, L'Harmattan.

Bulgakova Tatiana, 2008, "Revival of Christianity and Shamanism Among the Indigenous People of Siberia ", in Lardinois Olivier et Vermander Benoît, Shamanism and Christianity, Religious Encounters among Indigenous People of East Asia, Taipei, Taipei Ricci Institute, p. 145-162. 
Bulkeley Kelly, 2008, "The Impact of Cognitive Science on Religious Studies: A Revolution in the Making ", Religious Studies Review, 34/4, p. 239-246.

Cauquelin Josiane 1991, "Le chamanisme chez les Puyuma de Taiwan et son évolution sous l'impact de l'occupation japonaise ", Archipel, 4, p. 139-152.

-, 2004, The Aborigines of Taiwan. The Puyuma: From Headhunting to the Modern World, London, Routledge Curzon.

Chang Kwang-chich, 1983, Art, Myth, and Ritual: The Path to Political Authority in Ancient China, Cambridge, MA: Harvard University Press.

-, 1994, "Shang Shamans ", in Peterson W. J. (ed.), The Power of Culture: Studies in Chinese Cultural History, Hong Kong, The Chinese University Press.

-, 1999, "China on the Eve of the Historical Period », in Loewe-Edward Michael et Shaughnessy Edward L. (eds.), The Cambridge History of Ancient China, Cambridge, Cambridge University Press.

CHEN Mengjia, 1936, "Shangdai de shenhua yu wushu " (Mythes et techniques chamaniques à l'époque shang), Yangjing xuebao, 1936, 20, p. 486-576.

CHor Chungmoo, 1987, The Competence of Korean Shamans as Performers of Folklore, Diss., Indiana University.

CHor Ki-bok, 1995, «A Theology of Life ", Inculturation, Korea, 5 (3), Automne 1995, p. 2-4.

Clark Peter, 2006, New Religions in Global Perspective, Abirgdon, Routledge.

ChIng Julia, 1997a, Mysticism and Kingship in China: The Heart of Chinese Wisdom, Cambridge, Cambridge studies in religious traditions.

-, 1997b, "Son of heaven: Sacral kingship in ancient China ", T'oung Pao, 83, p. 3-41.

Collot Édouard, Hell Bertrand, 2011, Soigner les âmes. L'invisible dans la psychothérapie et la cure chamanique, Paris, Dunod.

Delaplace Grégory, 2009, L'invention des morts, sépultures, fantômes et photographie en Mongolie contemporaine, Paris, Centre d'Études Mongoles et Sibériennes - École pratique des hautes études, "Nord-Asie I ».

Donohue Mark, Denham Tim, 2010, "Farming and Language in Island Southeast Asia: Reframing Austronesian History ", Current Anthropology, 51 (2), p. 223-256.

Dulam Bumochir, 2010, « Degrees of Ritualization: Language Use in Mongolian Shamanic Ritual », Shaman, 18 (1-2), p. 11-42.

EICHHORN Werner, 1979, «Allgemeine Bemerkung über das Religiöse im alten China ", Oriens Extremus, 26 (1-2), p. 13-21.

Eliade Mircea, 1951, Le chamanisme et les techniques primitives de l'extase, Paris, Plon.

-, 1964, Shamanism: Archaic Techniques of Ecstasy, Bollingen Series $\mathrm{N}^{\circ}$ LxxvI, Princeton, Princeton University Press.

Frecksa Ede, Luna Luis Eduardo, 2007, "The Shamanic Healer: Master of Nonlocal Information? ", Shaman, 15 (1-2), p. 136-160.

Fu Yuguang, Meng Huiying, 1991, Manzu samanjiao yanjiu (Recherches sur le chamanisme mandchou), Pékin, Presses de l’Université de Pékin.

Fu Yuguang, WANG Honggang, 1995, Samanjiao nüshen (Déesses chamaniques), Shenyang, Presses populaires du Liaoning.

Fu Yuguang, 2000, Samanjiao lun (Sur le chamanisme), Shenyang, Presses populaires du Liaoning. 
FunG Jojo, 2008, "A World beyond the Threshold », in Lardinois Olivier et Vermander Benoît (eds.), Shamanism and Christianity: Religious Encounter among Indigenous Peoples of East Asia, Taipei, Taipei Ricci Institute, "Variétés sinologiques ", new series no 96, p. 29-49.

Gibson Todd, 1997, "Notes on the History of the Shamanic in Tibet and Inner Asia ", Numen, 4, p. 39-59.

HAMAYON Roberte, 1990a, La Chasse à l'Âme, esquisse d'une théorie du chamanisme sibérien, Nanterre, Société d'Ethnologie.

-, 1990b, "Le chamanisme, forme religieuse élémentaire ", Revue de l'Institut catholique de Paris, 34, avril-juin 1990, p. 33-55.

-, 1995, "Pour en finir avec la "transe" et "l'extase" dans l'étude du chamanisme ", Études mongoles et sibériennes, 26, "Variations Chamaniques 2 ", p. 155-189.

-, 1998a, "Chamanisme ", Paris, Encyclopedia universalis, t. 5, p. 303-306.

-, 1998b, «Le sens de "l'Alliance" religieuse. "Mari” d'esprit, "femme” de dieu ", Anthropologie et Sociétés, 22 (2), p. 25-48.

-, 2003, "Introduction à Chamanismes. Réalités autochtones, réinventions occidentales ", in Hamayon R. N. (éd.), Chamanismes, Revue Diogène, Paris, Quadrige / PUF, 2003, p. 7-54.

-, 2006, "L'idée de “contact direct avec des esprits” et ses contraintes d'après l'exemple de sociétés sibériennes ", Afrique \& histoire, 2006/2, vol. 6, p. 13-39.

Hell Bertrand, 1999, Possession et chamanisme, les maîtres du désordre, Paris, Flammarion, coll. "Champs".

Hopkins L. C., 1945, "The shaman or Chinese wu », JRAS, p. 3-16.

Hoppal Mihaly, 1992, "Pain in Shamanic Initiation ", in Siikala Anna-Leena et Hoppal Mihaly (eds.), Studies on Shamanism, Helsinki, Finnish Anthropological Society, p. $150-155$.

Hu Kuozhen, Ding Liwei, Zhan Changhui, 2008, Yuanzhumin wushu yu jiduzongjiao (Chamanisme aborigène et christianisme), Taipei, Kuangchi Cultural Enterprise.

Hu Tai-li, LIU Pi-chen (dir.), 2010, Taiwan yuanzhumin wushi yu yishi zhanbian (Chamanisme et performances rituelles des aborigènes de Taiwan), Taipei, Academia Sinica, Institut d'ethnologie.

Hu Tai-li, 2010, «Paiwan gulou nüwushi changjing de dangdai zhanyan » (La performance contemporaine des chants rituels par les chamanesses paiwan de Kulalao), in Hu Tai-li et Liu Pi-chen (dirs.), 2010, Taiwan yuanzhumin wushi yu yishi zhanbian (Chamanisme et performances rituelles des aborigènes de Taiwan), Taipei, Academia Sinica, Institut d'ethnologie, p. 23-68.

-, 2010, "Chants and Healing Rituals of the Paiwan Shamans in Taiwan ", Shaman, XVIII (1 \& 2), p. 43-54.

-, 2011, "Paiwan Shamanic Chants in Taiwan: Texts and Symbols ", Shaman, XIX (1 \& 2), p. 5-29.

Hubbard Timothy L., 2002, "Some Correspondences and Similarities of Shamanism and Cognitive Sciences: Interconnectedness; Extension of Meaning and Attribution of Mental States ", Anthropology of Consciousness, 13 (2), p. 26-45.

HumPHREY Caroline, ONON Urgunge, 1996, Shamans and Elders: Experience, Knowledge, and Power Among the Daur Mongols, Oxford, Clarendon Press, coll. "Oxford Studies in Social and Cultural Anthropology ". 
HutTon Ronald, 2001, Shamans: Siberian Spirituality and the Western Imagination, New York, Hambledon and London.

JiANG Xiangxun, 1995, Shenmi qinggong saman jisi (Cultes chamaniques secrets du Palais impérial sous les Qing), Shenyang, Presses populaires du Liaoning.

KeIghtley David, 1998 "Shamanism, Death, and the Ancestors. Religious Mediation in Neolithic and Shang China (ca. 5000-1000 BC) ", Asiatische Studien, 52.3 (1998), p. 763-831.

Kendall Laurel, 1991, An Initiation Kut for a Korean Shaman, video, Honolulu, University of Hawai'i Press.

-, 1996, "Initiating Performance: The Story of Chini, a Korean Shaman », in Laderman Carol et Roseman Marina (eds.), The Performance of Healing, New York, Routledge.

Kim Hogarth Hyun-Key, 2010, "The Korean Shamanistic Ritual and Psychoanalysis ", Shaman, XVIII (1 \& 2), p. 55-67.

KIRBy Ernest Th., 1975, Urdrama: The Origins of Theater, New York, New York University Press.

KISTER Daniel A., 1997, Korean Shamanist Ritual, Budapest, Akademiai Kiado.

-, 2010, Shamanic Worlds of Korea and Norheast Asia, Séoul, Jimoondang, "Korean Studies Series ", $\mathrm{n}^{\circ} 45$.

Kleinman Arthur, Sung Lilias H., 1979, "Why do Indigenous Practitioners Successfully Heal ", Social Science and Medicine, 13B, p. 7-26.

Kosa Gabor, 2000, "In Search of the Spirits": Shamanism in China Before the Tang Dynasty. Part One », Shaman, VIII (2), p. 131-179.

-, 2007, "The Jurchen Shamaness. An Analysis of the First Written Reference to the Word "Shaman" ", Shaman, XV (1-2), p. 117-128.

KuIPERs Joel C., 1990, Power in Performance: The Creation of Textual Authority in Weyewa Ritual Speech, Philadelphia, University of Pennsylvania Press.

KuRTi Laszlo, 2007, «Language, Symbol and Dance: An Analysis of Historicity in Movement and Meaning ", 2 (1-2), p. 23-82.

Lansing Stephen, J., Cox Murray P., De Vet Therese A., Downey Sean S., Hallmark Brian, Sudoyo Herawati, 2011, "An ongoing Austronesian Expansion in Island Southeast Asia ", Journal of Anthropological Archaeology, 30 (3), p. 262-272.

Lasimbang Anne, 2008, "Survival of Shamanism among the Kadazandusun of North Borneo; A Christian Women's Perspective ", in Lardinois Olivier et Vermander Benoît (eds.), Shamanism and Christianity: Religious Encounter among Indigenous Peoples of East Asia, Taipei, Taipei Ricci Institute, "Variétés sinologiques », new series n 96, p. 19-27.

LeFeuvre Jean, 1997, "Grands et petits territoires ", in Gernet Jacques et Kalinowski Marc (éds.), En suivant la voie royale. Mélanges en hommage à Léon Vandermeersch, Paris, École d'Extrême-Orient, Études thématiques 7, p. 45-49.

LÉvi-STRauss Claude, 1949, "Le sorcier et sa magie ", Les Temps Modernes, 4 (41), p. 3-24 (reproduit in Anthropologie structurale), Paris, Plon, 1958, p. 191-212.

LIU Housheng, 1993, Qingdai gongting saman jisi (Cultes chamaniques à la cour des Qing), Changchun, Presses littéraires et historiques du Jilin.

LIu Pi-chen, 2011, "Encounters with Deities for Exchange: The Performance of Kavalan and Amis Shamanic Chants ", Shaman, 19 (1-2), p. 107-128. 
-, 2003, « Missionnaires hommes VS Mtiu (femmes chamanes) : transformation de l'identité et du pouvoir des femmes chez les Kavalan, Austronésiens de Taiwan ", Histoire et Anthropologie ASIES 2, p. 49-62.

-, 2007, "Cerf-amant, coq-mari et femmes chamanes - disjonction des sexes chez les Kavalan (Taiwan) ", in Mathieu Nicole-Claude (éd.), Une maison sans fille est une maison morte - La personne et le genre en sociétés matrilinéaires et/ou uxorilocales, Paris, Édition de la Maison des Sciences de l'Homme, p. 385-414.

MA Changshou, 2006, Liangshan luoyi kaocha baogao (Rapport d'enquête sur les Lolos \{Yi\} de Liangshan), 2 volumes, Chengdu, Société de publication Bashu du Sichuan.

MA Erzi, 2009, "Jianshu liangshan yizu shoulie shenghuo ji shoulie fagui » (Sur la vie et les règles de chasse chez les Yi de Liangshan), Liangshan minzu yanjiu, p. 21-25.

-, 2010, "Liangshan yizu sangshi guijü ji qi bianqian " (Règles funéraires chez les Yi de Liangshan et leurs transformations), Liangshan minzu yanjiu, p. 47-54.

McCoy Brian F., 2008, "Maparn: Traditionalist Health and Healing in a Western and Christian world ", in Lardinois Olivier et Vermander Benoît (eds.), Shamanism and Christianity: Religious Encounter among Indigenous Peoples of East Asia, Taipei, Taipei Ricci Institute, "Variétés sinologiques », new series no 96, p. 195-212.

McFarlane Alan, 2007, Japan Through the Looking Glass: Shaman to Shinto, London, Profile Books.

Major John S., 1978, "Research Priorities in the Field of Chu Religion ", History of Religions, 17, p. 226-243.

-, 1999, "Characteristics of Late Chu Religion ", in Cook Constance A. et Major John S. (eds.), Defining Chu, Image and Reality in Ancient China, Honolulu, University of Hawai'i Press.

Marshall Alison, 2003, «Engendering Mediumship: When Youths Performed the Rain Dances in Han Dynasty China ", Studies in Religion, 32 (3), p. 83-100.

Matthieu Rémi, 1987, « Chamanes et chamanisme en Chine ancienne ", L'Homme, 101, février-mars 1987, p. 10-34.

-, 2009, "Les Wu : fonctions, rites et pouvoirs de la fin des Zhou au début des Han », in Lagerwey John (dir.), Religion et société en Chine ancienne et médiévale, Paris, Éditions du Cerf, Institut Ricci, p. 218-304.

MENG Huiying, 2000, Zhongguo beifang minzu samajiao (Le chamanisme des ethnies $d u$ Nord de la Chine), Beijing, Presses de l'académie des sciences sociales.

-, 2010, "Zai ji zu duili de sikao zhong renshi samanjiao » (Comprendre le chamanisme à partir de perspectives opposées), Zongjiao renleixue (Anthropologie religieuse), $\mathrm{n}^{\circ} 2$, p. 313-334.

Merli Laetitia, 2004, De l'ombre à la lumière, de l'individu à la nation : renouveau chamaniste en Mongolie post-communiste, Paris, EHESS, thèse de doctorat d'anthropologie sociale et culturelle.

Métraux Alfred, 1967, Magies et Religions d'Amérique du Sud, Paris, Gallimard.

Mitrani Philippe, 1982, "Aperçu critique des approches psychiatriques du chamanisme ", L'Ethnographie, 88, p. 241-257.

Mitsuda Yayoi, 2010, "Xiangshengma, wenben yu yishi biaoyan : dangdai shaozu de shengcun celüe » (Chamanesses, textes et performances rituelles : stratégies de survie des Shao à l'époque contemporaine), in Hu Tai-li et Liu Pi-chen (dirs.), 2010, Taiwan yuanzhumin wushi yu yishi zhanbian (Chamanisme et performances rituelles des aborigènes de Taiwan), Taipei, Academia Sinica, Institut d'ethnologie, p. 467-503. 
-, 2011, "Cultural Memory in Shamanic Chants: A Memory Storage Function of Thao Shamans ", Shaman, XIX (1 \& 2), p. 129-152.

MuegGler Erik, 2001, The Age of Wild Ghosts, Memory, Violence and Place in Southwest China, Berkeley, University of California Press.

Névot Aurélie, 2008, Comme le sel, je suis le cours de l'eau, le chamanisme à écriture des Yi du Yunnan (Chine), Nanterre, Société d'ethnologie.

Noll R., 1983, "Shamanism and Schizophrenia: A State-Specific Approach to the "Schizophrenia Metaphor” of Shamanic States ", American Ethnologist, 10, p. 443-459.

OrTnER Sherry, 1998, "The Case of the Disappearing Shamans, or No Individualism, No Relationalism ", in Skinner Debra, Pach III Alfred et Holland Dorothy (eds.), Selves in Time and Place. Identities, Experience, and History in Nepal, Lanham, Boulder, New York, Oxford, Rowman \& Littlefield Publ. Inc, p. 239-267.

PAng Tatiana A., 2007, "The Kun-ning-gung Palace in Peking: The Manchu Dynasty's Shaman Centre in the "Forbidden City" ", Shaman, 2007-1 (1-2), p. 71-86.

Pelliot Paul, 1913, "Sur quelques mots d'Asie Centrale attestés dans les textes chinois ", Journal Asiatique, XI, I, p. 451-469.

PERRIN Michel, 1992, Les praticiens du rêve. Un exemple de chamanisme, Paris, PUF.

QIU Yunfang, 2004, Zuling, shangdi yu chuantong - jiduchanglao jiaohui yu Truku ren de zongjioa bianqian (Esprits ancestraux, Dieu et la tradition - L'église presbytérienne et les changements religieux chez les Truku), Taipei, thèse soutenue dans le département d'anthropologie de l'université nationale de Taiwan.

QIU Xigui, 1983-1985, " On the Burning of Human Victims and the Fashioning of Clay Dragons in Order to Seek Rain as Seen in the Shang Dynasty Oracle Bone Inscriptions ", translated by Vernon K. Fowler, Early China, 9-10, p. 301-303.

Rudolph Michael, 2008, Ritual Performances as Authenticating Practices, Cultural Representations of Taiwan's Aborigines in Times of Political Changes, Berlin, LIT Verlag, «Performanzen ", vol. 14.

SALAdin D'Anglure Bernard, 1986, "À l'ombre du troisième sexe. Reproduction de la vie, transe et chamanisme chez les Inuit de l'Arctique central canadien ", in Transe, chamanisme et possession, Actes des $2^{e}$ rencontres internationales sur la fête et la communication, Nice, p. 111-115.

-, 1992, "Le "troisième" sexe ", La Recherche, 245 (23), p. 836-844.

SAles Anne de, 1991, Je suis né de vos jeux de tambours, Nanterre, Société d'ethnologie. Samuel Geoffrey, 1993, Civilized Shamans: Buddhism in Tibetan Societies, Washington and London, Smithsonian Institute Press.

-, 2010, "Healing, Efficacy and the Spirits ", Journal of Ritual Studies, 24 (2), p. 7-20.

SCHAFER Edward, 1951, "Ritual Exposure in Ancient China ", Harvard Journal of Asiatic Studies, 1-2, p. 130-184.

Sharma Arvind, 2006, A Primal Perspective on the Philosophy of Religion, Dordrecht, Springer.

Shirokogoroff Sergei M., 1935, Psychomental Complex of the Tungus, London, Kegan Paul, Trench, Trubner.

SiIKALA Anna-Leena, 1978, The Rite Technique of the Siberian Shaman, Helsinki, Academia Scientiarum Fennica.

SiIKAla Anna-Leena, Ulyashev Oleg, 2003, «Landscape of Spirits: Holy Paces and Changing Rituals of the Northern Kanty ", Shaman, 11 (1-2), p. 149-178. 
STARY Giovanni, 2007, " "Praying in the Darkness": New Texts for a Little-Known Manchu Shamanic Rite ", Shaman, 1 (1-2), p. 87-104.

Stepanoff Charles, 2004, "Chamanisme et transformation sociale à Touva ", Études mongoles et sibériennes, centrasiatiques et tibétaines, 35, p. 155-183.

Stroeken Koen, 2011, "Questioning Cognitive and Interpretive Takes on Ritual ", Anthropological Theory, 11 (3), Sept. 2011, p. 355-372.

Tambiah Stanley J., 1979, "A Performative Approach to Rituals ", Proceedings of the British Academy, 1979, 65, p. 113-169.

ToNG Enzhen, 1930, «Shi wu » (Une interprétation du caractère wu), Yanjing xuebao, $1930, \mathrm{n}^{\circ} 7$, p. 1327-1345.

Tu Baikui 1997, "Shi wu » (Une interprétation du caractère wu), Huaxia kaogu, 1997-1, p. 89-92.

VAzeILles Danièle, 1990, Les chamanes maîtres de l'univers. Persistance et exportations $d u$ chamanisme, Paris, Éditions du Cerf, coll. «Bref ».

VAn Gennep Arnold, 1903, "De l'emploi du mot "Chamanisme” ", Revue de l'histoire des religions, 47 , p. 51-57.

Von Falkenhausen Lothar, 1995, "Reflections on the Political Role of Spirit Mediums in Early China: The Wu Officials in the Zhouli», Early China, 20, p. 279-300.

-, 2006, Chinese Society in the Age of Confiucius (1000-250 BC): The Archaeological Evidence, Los Angeles, Corsen Institute of Archaeology / University of California.

Waley Arthur, 1955, The Nine Songs: A Study of Shamanism in Ancient China, London, Allen \&Unwin.

WANG Hui, 2001, Shangdai wenhua bijioa yanjiu (Recherches comparées sur la culture de la dynastie Shang), Beijing, Presses populaires de Chine.

YANG Boda, 2005, «Wu-yu-shen fanlun (Shaman, jade et dieu : une théorie générale) », Zhongyuan wenwu, 4, p. 63-69.

Zhaо Zhizhong, 2008, Zhongguo samaniiao (Le chamanisme chinois), Xining, Presses populaires du Qinghai. 
